\title{
A Classroom Experiment on the Causes and Forms of Bounded Rationality in Individual Choice
}

Anna Rita Bennato, Adrian Gourlay and Chris M. Wilson*

School of Business and Economics, Loughborough University, Loughborough, Leicestershire, UK

\begin{abstract}
Bounded rationality is a key concept with implications across all areas of economics. To help students better understand the nature, causes and forms of bounded rationality in individual choice, we present a flexible classroom experiment. Beyond providing students with some first-hand evidence of bounded rationality, the experiment shows how a range of factors can prompt bounded rationality and illustrates how it can manifest itself in the form of different behaviours. The experiment can be conducted with any number of students and takes approximately 30 minutes to conduct, with a further 10-20 minutes to reflect and debrief.
\end{abstract}

JEL Classification: A22; D90; D80.

Keywords: Bounded rationality; classroom experiment; choice; heuristics; information search.

*Corresponding author email: c.m.wilson@lboro.ac.uk 


\section{INTRODUCTION}

Under standard neo-classical theory, individuals are always able to perfectly gather and process any available information in order to select the option that maximises their utility. However, in practice, individuals tend to be constrained in their time and cognitive abilities. Hence, in the presence of such constraints, rather than carefully considering all the relevant information and working out the 'correct' option, individuals may find it optimal to use an approximation. Better understanding such bounded rationality will lead to more accurate economic models with the capability of offering improved policy advice. As such, bounded rationality is an active research area with implications across the whole spectrum of economics. ${ }^{1}$

To help students comprehend the nature, causes and forms of bounded rationality, this paper presents a flexible classroom experiment. Beyond providing students with some first-hand evidence, the experiment shows how a range of factors can cause bounded rationality and how it can manifest itself in different forms. The experiment can be conducted with any number of students and takes approximately 30 minutes to conduct, with a further 10-20 minutes to reflect and debrief. It is best suited to an introductory/intermediate microeconomics course or an introductory course on behavioural economics, in the context of individual decision-making or consumer choice.

Even trivial decisions can be mentally challenging. For instance, Kahneman (2011, p.20) asks readers to imagine being asked to multiply 17 by 24 . Moreover, many life decisions are significantly complex. As an example, Benartzi and Thaler (2007) outline the huge difficulties that individuals face when trying to choose their optimal pension plan. When faced with such demanding tasks, instead of calculating the fully rational decision, individuals may choose to optimally conserve cognitive resources by using an approximation. For example, individuals may only pay attention to some of the relevant information - Simon's (1955) concept of 
'satisficing' suggests that individuals will only gather and process information when the marginal net benefit of doing so is positive. Alternatively, even if individuals consider all the information, they may still make their selection decisions with 'rules of thumb' or 'heuristics' (Gilovich et al 2002). Such instances of bounded rationality are known to be more likely when the decision options are complex or numerous, and when individuals are inexperienced, fatigued, or under time pressure.

However, obtaining evidence of bounded rationality is inherently difficult due to a measurement issue whereby any potential evidence of a boundedly rational choice can be alternatively explained by the individual's unobserved preferences. Did an individual choose an 'incorrect' option because they were using an optimal approximation or because they just preferred that option?

Caplin et al (2011) introduce a research methodology to overcome this measurement issue by asking their experimental subjects to choose the highest value option from a list of different mathematical calculations. As the subjects should have no other underlying preferences amongst such options, any selection of an 'incorrect' answer can be best interpreted as resulting from bounded rationality. In addition, Caplin et al (2011) also introduce a second innovation. By using specialist software, subjects are given an unknown random time deadline for their final answer in a way that prompts them to continuously reveal their current best decision. This allows the authors to conduct a rich analysis of individuals' search processes and decisionmaking in order to formally discriminate between different aspects of bounded rationality. In particular, they show how the data is most consistent with an explanation of 'satisficing' where individuals do not consider the entire set of options, rather than an explanation where individuals are fully informed but use approximations in their calculations and selection decisions. 
Our teaching experiment utilises a simplified and accessible form of Caplin et al's experiment to help instructors demonstrate the nature, causes and forms of bounded rationality. By adopting Caplin et al's first innovation, we measure bounded rationality by asking students to choose from lists of mathematical calculations. However, rather than using Caplin et al's second innovation by continuously monitoring students' choices, we simply ask the students for their final answers at the end of a known time limit. While this reduces our ability to formally discriminate between different aspects of bounded rationality, it enables a broad study within a practical classroom setting. In particular, by varying the number and complexity of the calculations, the experiment can i) analyse how such factors affect bounded rationality, and ii) illustrate how bounded rationality can manifest itself in different forms. For instance, students may exhibit a 'primacy effect', where they are more likely to select options towards the top of the list, or a 'left-digit bias', where they pay relatively more attention to digits towards the start of each calculation. ${ }^{2}$

Classroom experiments are well documented to be a valid instrument to engage students and improve their learning experience (e.g. Emerson and Taylor 2004, Durham et al 2007, Dickie 2010, Cartwright and Stepanova 2012). However, perhaps due to the measurement issue described above, there are very few classroom experiments on the topic of bounded rationality. Moreover, the existing experiments focus on some broader aspects of bounded rationality that are very different to the topic of our paper - Dupont and Durham (2018) consider Bayesian reasoning in the Monty Hall Dilemma, Alba-Fernández et al (2010) study learning and convergence in strategic Beauty Contest Games, and Beckman et al (2011) use a questionnaire approach to introduce students to some concepts within wider behavioural economics. 


\section{THE EXPERIMENT}

The experiment consists of twelve multiple choice questions. In each question, the students are requested to identify the option which offers the highest value where each option involves a short calculation based on the addition and subtraction of some random numbers. To make the choice process more challenging, the numbers are written in a text format, rather than a numeric format. An example question is provided in Figure 1 below.

[Figure 1 about here]

While the format of such questions may seem abstract initially, its merits are two-fold. First, and most importantly, it helps to cleanly tackle the measurement issue noted in the introduction - students should have no underlying preferences amongst the different options. Second, such a task is related to some common economic situations. For instance, choosing from a list of items with numerical content has some similarities to searching for a supplier on a comparison site, comparing options from a set of search results or website, and investigating listings of financial products, job opportunities, or accommodation vacancies.

In more detail, the experiment's twelve multiple choice questions are divided into four rounds of three questions. Within each round, there are three different types of questions that we refer to as Simple 4, Simple 8 and Complex 4. Hence, in each round, there is one Simple 4 question, one Simple 8 question and one Complex 4 question, and overall, the experiment contains four Simple 4 questions, four Simple 8 questions, and four Complex 4 questions.

The first type of question, Simple 4, is our benchmark format. It has four options where each option involves two mathematical operations, as consistent with Figure 1. The second and third types of questions vary this format to further test the causes of bounded rationality. The second type, Simple 8, has the same form of options as Simple 4, but involves eight rather than four options, as shown in Figure 2. 
[Figure 2 about here]

The third type, Complex 4, has four options but each option involves a more difficult calculation with three rather than two mathematical operations, as illustrated in Figure 3.

[Figure 3 about here]

Students' responses to the twelve questions are best collated anonymously using any standard electronic voting system that uses clickers or students' own web-enabled devices (i.e. cell phones). This approach is useful because it allows the instructor to i) control the time available for each question, and ii) collate the experimental data efficiently with a potentially large number of students. ${ }^{3}$ However, if such technology is not available, students' responses can be alternatively collated on paper. ${ }^{4}$

For each question, a time limit of twenty seconds is strictly applied. After some careful trials, this time length is viewed as being sufficiently long to allow students enough time to have a good effort at each question, but short enough to put the students under some pressure. To make students aware of the time constraint, a countdown clock is presented on the screen, and a final warning is provided with five seconds remaining. Students are permitted to use rough paper to assist with any calculations during the time limit.

If an individual student does not submit an answer to a question within the allotted time, no default answer is applied. Instead, it is simply marked down as a non-response. Later results will record the response rate for each question, and the proportion of responses that chose each option. Monetary incentives are not viewed as necessary to elicit responses from students. From our own experience of using the experiment with large groups of students where monetary incentives have not been feasible, excellent results have still been obtained by encouraging the students to answer the questions to the best of their abilities for the good of the teaching session. $^{5}$ 
At the beginning of the experiment, the instructor reads through the instructions. (A full set of instructions is provided in the Appendix, together with details of the actual questions and an explanation of how they were created.) To ensure the effectiveness of the experiment, it is important to remind students to submit their best answer by the end of the time limit even when they are not sure of their own response. In order to be certain that students have clearly understood the instructions and the tasks, the instructor then conducts a practice session. Within the practice sessions, the students are asked to answer three example questions, involving one of each type. After each practice question, the instructor discusses the correct answer and uses the electronic voting system to show the proportion of students that picked each option. This helps the students to further understand the format of the experiment and the tasks.

The instructor then moves on to the twelve questions within the main experiment. In order to conserve students' attention, the answers and responses are not revealed at the end of each question. Instead, a short 1-2 minute break is provided after each round to allow the students to chat with their neighbours and to help maintain later concentration.

In our experience, we have found one potential pitfall with the experiment. When using the electronic voting system, it is possible for the instructor to accidentally advance the slides too quickly and thereby close a question's response period before the twenty second period has elapsed. If this happens, the question is void. To resolve this, we successfully continued the experiment and only used the data from the three unaffected rounds with no major impact on the results.

After the experiment, the instructor should help the students to reflect on their experiences and provide an explanation of the experimental results. This can be done in a number of ways. However, we typically proceed as follows. 
First, immediately after the experiment and before any instructor-led discussion about the hypotheses, results or explanations, we ask the students to reflect on their choices. We ask them about which types of questions were the hardest, which factors influenced their decisionmaking approach, whether they used any specific rules of thumb, and how such rules of thumb may have led them to select certain types of options more than others. Some example reflection text, questions and typical answers are provided in the Appendix.

Depending on the size of group and the length of session, these reflections may take a number of formats and be fed back to the instructor to form a summary discussion in a number of ways. In our larger group sessions with limited time, we ask the students to reflect by having a short discussion with their neighbours and then ask for volunteers to share their thoughts to the class. Despite the large group, we find that a sufficient number of students are willing to make contributions that are consistent with the typical answers listed in the appendix. In total, this approach of reflection and discussion takes 10-20 minutes. However, in other contexts, there may be more opportunities to allow students to write down their reflections more formally which is argued to lead to a better learning experience (e.g. Cartwright and Stepanova 2012).

Second, after the teaching session, the instructor will have more time to collate and tabulate the results from the experiment. These can be emailed to students in the form of a short report and/or revisited with some brief time within the next teaching session. In either case, the instructor is recommended to summarise the main points of the experiment and to present the results. As outlined in the next two sections, the results can be presented in conjunction with the hypotheses to show the potential evidence of i) bounded rationality, ii) the factors affecting bounded rationality, and iii) some typical forms of bounded rationality. The instructor can also use this opportunity to highlight the importance of bounded rationality to economics and to explain the reasoning behind the experiment. 


\section{HYPOTHESES}

\section{The Existence of Bounded Rationality}

Under bounded rationality, we would expect some students to depart from the fully rational prediction. In particular, when faced with active constraints in their time and/or cognitive abilities, students may optimally use an approximation. However, such approximations may not always lead to the 'correct' answer. Hence, we would expect the percentage of incorrect answers to be larger than zero.

\section{The Causes of Bounded Rationality}

We also expect the percentage of incorrect answers to be larger in situations where bounded rationality is more likely. In particular, it is well known that individuals are likely to face higher costs of mental processing i) when the options are more complex, ii) when the options are more numerous, iii) when they are making decisions for the first time, iv) when they are fatigued, or v) when they are under time pressure. In such situations, it is more likely that individuals will optimally conserve their cognitive resources, and so it is less likely that they will select the correct answer. Thus, relative to the responses in the Simple 4 questions, we would expect the percentage of incorrect answers to be larger when i) there are more options (in Simple 8), and ii) the options are more difficult (in Complex 4). We may also expect the percentage of incorrect answers to be higher in the early rounds of the experiment when the students are inexperienced and/or the later rounds of the experiment when the students may be fatigued.

\section{The Forms of Bounded Rationality}

The forms of bounded rationality will depend upon the decision-making approach chosen by the students. Many of these derive from Simon's (1955) central concept of satisficing where an individual optimally processes information only if the marginal return is positive. Some typical forms could include the following: 
- A left-digit bias refers to a case where a student is relatively more inclined to select an option that starts with a higher number, e.g. nine plus two minus three. This is consistent with a student starting a calculation from the left-hand-side but finding it too costly to complete all the mathematical operations within the calculation. Similarly, a right-digit bias refers to a case where a student gives disproportionate attention to the value of the number at the end of each calculation, (for example see Lacetera et al 2012). ${ }^{6}$

- $\quad$ Primacy effects refer to cases where a student is more inclined to pick higher-positioned options, such as option A. This can be consistent with a student inspecting the options sequentially from the top downwards and then finding it too costly to calculate all the lower options. Recency effects refer to cases where a student is more inclined to pick lowerpositioned options, such as option D (for four options) or option $\mathrm{H}$ (for eight options). These can be consistent with a similar explanation, but where a student inspects the options sequentially from the bottom-up, (for example, see Meredith and Salant 2013).

\section{EXAMPLE RESULTS}

In this section we report some example results from an occasion where we conducted the experiment within an intermediate microeconomics module with around 120 undergraduate students. The Appendix provides some tips for instructors when calculating their own set of results.

We first report some summary statistics in Table 1 . This presents the average response rate, and the average percentage of incorrect answers from the responses received. Table 1 also splits these results by round, and by question type (Simple 4, Simple 8 and Complex 4). The qualitative patterns described within Table 1 are very reliable across the different occasions that we have run the experiment.

[Insert Table 1 about here] 


\section{Response Rate}

Although some students did not always provide an answer within the time limit, the average response rate was consistently high throughout the experiment.

\section{The Existence of Bounded Rationality}

Students provided an incorrect answer $23 \%$ of the time across the whole experiment. This is consistent with bounded rationality, and inconsistent with full rationality where students would be expected to never select a wrong answer. Such behaviour is also inconsistent with the students picking the options randomly without engaging with the experiment as they would then be expected to select a wrong answer $75 \%$ of the time for 4-option questions and $88 \%$ of the time for 8-option questions.

\section{The Causes of Bounded Rationality}

Consistent with bounded rationality being more likely when the number of options is larger, we can see that the Simple 8 questions led to a substantially higher percentage of incorrect responses relative to the Simple 4 questions. Similarly, as consistent with bounded rationality being more likely when the options are more difficult, the Complex 4 questions also led to a higher percentage of incorrect responses relative to the Simple 4 questions. Finally, as consistent with the predicted effects of inexperience and fatigue on bounded rationality, the average proportion of incorrect answers peaked in the first and last rounds.

\section{The Forms of Bounded Rationality}

We now move on to considering some specific forms of bounded rationality observed within the data. In a typical session, the results are often consistent with one or more forms of bounded rationality. However, the exact form can vary across sessions and the results can be more 
variable than those previously presented in Table 1 . We now discuss the potential evidence of a left/right digit bias or a primacy/recency effect in our example results.

i) Left/Right Digit Bias: As consistent with a left-digit and/or a right-digit bias, some students appear to be more influenced by the value of the digits at the start and end of each calculation, relative to the value of the digit in the middle. This is supported by Table 2 which considers how students' incorrect choices were influenced by the specific digits in each option. In particular, Table 2 reports the correlation between the number of students who selected a given incorrect option and the value of the options' different digits. ${ }^{7}$ The results show that students' incorrect choices were positively correlated with the value of the first digit in the option (which was always added in the calculation), and negatively correlated with the value of the last digit in the option (which was always subtracted in the calculation). However, students' incorrect choices were less correlated with the value of the middle digit in each option (which was always added in the calculation), suggesting that the middle digit was given less attention in students' calculations.

\section{[Insert Table 2 about here]}

ii) Primacy/Recent Effects: In this particular set of results, there was no strong evidence of primacy or recency effects. On average, students were not disproportionately inclined to select options in top position or bottom position. In principle, this can be analysed in a number of ways. However, the cleanest and easiest way is to consider the Simple 4 and Complex 4 questions where students only faced four options, A-D. Absent any primacy or recency effects, we would expect students' choices to be distributed symmetrically across the four options because the answers were evenly allocated across each option. Table 3 shows that this is consistent with the data. However, there is some suggestions of a small primacy effect as option A was selected a little more frequently than the other options. This is also consistent with the 
reflection discussions after the experiment, where students often state they had inspected the options in a consistent descending (or ascending) order.

[Insert Table 3 about here]

\section{CONCLUSIONS}

Bounded rationality is an everyday issue, and a key active area in economics. However, there have been very few classroom experiments on the topic. Our experiment illustrates how a range of factors can cause bounded rationality and how bounded rationality can manifest itself in different forms. The experiment can be conducted efficiently with very large groups and takes 40-50 minutes to conduct and debrief.

The experiment appears to be very popular with students. After surveying our example cohort from an intermediate microeconomics course, we received the following results with a response rate of approximately $50 \%$. Compared to a normal lecture, $83 \%$ of students thought the experiment was more fun, $63 \%$ believed that it gave them a relatively deeper understanding, and $84 \%$ believed that it had prompted them to be relatively more interested in bounded rationality and behavioural economics. Some individual comments from the survey included the following: "It captured everyone's attention and helped to give us a deeper understanding rather than just giving us results and us not being able to interpret where the information came from", "It was very engaging and allowed us to gain a deeper understanding which would be difficult to do during a normal lecture" and "...I feel like this experiment helped explain it all in more depth - hence making it more interesting as there was an example to follow".

To extend the experiment, instructors could vary the time limit across questions in order to analyse the effects of time pressure as an additional cause of bounded rationality. In addition, to mirror the second innovation by Caplin et al (2011), instructors could try to measure students' choices in real-time by asking students to self-document their decision process. 


\section{NOTES}

1. For key references, see Simon (1955), Payne et al (1993), Gilovich et al (2002), and Sims (2003). For useful surveys and popular treatments, see Conlisk (1996), DellaVigna (2009), Kahneman (2011) and Thaler (2015).

2. For example references about such effects, see Meredith and Salant (2013) and Lacetera et al (2012) respectively.

3. For more on the value of using clickers in economics classes, see Salemi (2010).

4. Under a paper-based approach, instructors would provide each student with an answer sheet to record their responses at the start of the experiment, and tailor the experiment's instructions accordingly. To make the processing of such answer sheets as efficient as possible after the session, instructors could use optical mark recognition (OMR) as commonly used for multiple-choice tests and surveys.

5. For more on the use of monetary incentives in experiments, see Thaler (2015, p.4749) and Loewenstein (1999). See Rousu et al (2015) for monetary incentives in classroom experiments.

6. Strictly speaking, digit biases typically refer to calculations in numeric format, rather than in the written format used within our experiment. It would be interesting to compare how students behave across the different formats in future research.

7. These results also remain qualitatively robust if one calculates the correlations across all correct and incorrect options rather than across only the incorrect options. However, the presented approach has the advantage of not being unduly influenced by the format of the correct options. 


\section{REFERENCES}

Alba-Fernández, V., P. Brañas-Garza, F. Jiménez-Jiménez, and J. Rodero-Cosano. 2006. Teaching Nash equilibrium and dominance: a classroom experiment on the beauty contest. Journal of Economic Education 37(3): 305-322.

Beckman, S., L. Chen, G. DeAngelo, W.S. Smith, and X. Zhang. 2011. Microeconomics and psychology. Journal of Economic Education 42(3): 255-269.

Benartzi, S. and R. Thaler. 2007. Heuristics and biases in retirement savings behavior. Journal of Economic Perspectives 21(3): 81-104.

Caplin, A., M. Dean, and D. Martin. 2011. Search and satisficing. American Economic Review 101(7): 2899-2922.

Cartwright, E., and A. Stepanova. 2012. What do students learn from a classroom experiment: Not much, unless they write a report on it. Journal of Economic Education 43(1): 48-57.

Conlisk, J. 1996. Why bounded rationality? Journal of Economic Literature 34(2): 669-700.

DellaVigna, S. 2009. Psychology and economics: evidence from the field. Journal of Economic Literature 47(2): 315-72.

Dickie, M. 2010. Do classroom experiments increase learning in introductory microeconomics? Journal of Economic Education 37(3): 267-288.

Dupont, B., and Y. Durham. 2018. Let's make a deal in the classroom: Institutional solutions to the Monty Hall Dilemma. Journal of Economic Education 49(2): 167-172.

Durham, Y., T. McKinnon, and C. Schulman. 2007. Classroom experiments: Not just fun and games. Economic Inquiry 45(1): 162-78. 
Emerson, T., and B. Taylor. 2004. Comparing student achievement across experimental and lecture-oriented sections of a principles of microeconomics course. Southern Economic Journal 70(3): 672-93.

Gilovich, T., D. Griffin, and D. Kahneman. 2002. Heuristics and biases: The psychology of intuitive judgment. Cambridge: Cambridge University Press.

Kahneman, D. 2011. Thinking, fast and slow. London: Penguin Books.

Lacetera, N., D. G. Pope, and J. R. Sydnor. 2012. Heuristic thinking and limited attention in the car market. American Economic Review 102(5): 2206-2236.

Loewenstein, G., 1999. Experimental economics from the vantage-point of behavioural economics. The Economic Journal 109: F25-34.

Meredith, M., and Y. Salant. 2013. On the causes and consequences of ballot order effects. Political Behavior, 35(1): 175-197.

Payne, J. W., J. R. Bettman, and E. J. Johnson. 1993. The adaptive decision maker. Cambridge: Cambridge University Press.

Rousu, M.C., J. R. Corrigan., D. Harris., J. K. Hayter, S. Houser., B. A. Lafrancois., O. Onafowora., G. Colson., and A. Hoffer. 2015. Do monetary incentives matter in classroom experiments? Effects on course performance. Journal of Economic Education 46(4): 341-349.

Salemi, M.K. 2010. Clickenomics: Using a classroom response system to increase student engagement in a large-enrollment principles of economics course. Journal of Economic Education 40 (4): 385-404.

Simon, H. A. 1955. A behavioral model of rational choice. Quarterly Journal of Economics 69(1): 99-118. 
Sims, C. A. 2003. Implications of rational inattention. Journal of Monetary Economics 50(3): 665-690.

Thaler, R.H. 2015. Misbehaving: The making of behavioral economics. New York: WW Norton. 


\begin{abstract}
APPENDIX
In this appendix, we provide some extra "how-to" instructions and offer some additional resources to assist instructors.

\section{Tips for instructors when planning the experiment}

To prepare the experiment, an instructor must first decide how they will collect the students' responses. As explained in Section 2, we recommend using a clicker technology, but paperbased alternatives are also possible. An instructor can then use the material in this appendix to plan the session including the practice questions, the experiment, the reflection session debrief, and the construction of the results.
\end{abstract}

Some instructors may wish to develop their own questions rather than using the ones provided below. If so, we would offer the following piece of advice from our experience. To be able to perform a clean analysis of the four-option questions, it is useful to symmetrically distribute the answers across the options A-D. For instance, in our four Simple 4 and Complex 4 questions below, the answer is placed in each position exactly once. Under full rationality, one should then expect students' aggregate responses to be symmetrically distributed across the positions. This contrasts to the predictions under primacy/recency effects, where students' responses may be focussed towards the top or bottom of the list, respectively.

\title{
Initial instructions to students
}

"In the main experiment, we will go through twelve questions where each question will have a strict time limit. Responses are anonymous. Feel free to use a rough piece of paper to help you answer the questions. Please respond with your best answer by using your cell-phone (or clicker) (or by recording it on the provided answer sheet). Some of the questions are deliberately hard. If you can't work out the best answer, then it's important that you still give 
it your best educated guess. But please take it seriously and don't give up - otherwise, we won't be able to learn from the experimental results in the later lecture."

\section{Instructions to students about practice questions}

"We will now have a practice of 3 types of questions. You are aiming to select the option that maximises your payoffs (with the highest value). When we do it for real, there will be a time limit of 20 seconds per question. The first type of question has four options. The second type of question has eight options. The last type of question has four options but where each option is an extra calculation step."

\section{Practice questions for students}

The practice questions for the three types of questions were provided as the examples earlier in the text of the main paper - see Figures 1-3.

\section{Further instructions to students}

"We'll now start the experiment properly. There are 12 questions. There is a strict time limit of 20 seconds per question. There is a countdown clock and I will give you a 5 second warning as the time runs out. To help the experiment, please try hard to give an answer each time. If you don't know the answer or if you are running out of time, then please give your best guess before the time runs out."

\section{Actual questions for instructors}

To ease presentation, we now report the details of each question with its numbers in a numeric format rather than in the text format used in the experiment. To assist in the construction of later results, we also highlight the correct answer in bold. 
The four Simple 4 questions use the same format as that given in figure 1 together with the following numbers:

\begin{tabular}{llllllllllll}
\hline Q1 & 0 & plus & 1 & minus & 3 & Q4 & $\mathbf{8}$ & plus & $\mathbf{5}$ & minus & $\mathbf{7}$ \\
& 7 & plus & 3 & minus & 5 & & 3 & plus & 4 & minus & 3 \\
& $\mathbf{8}$ & plus & $\mathbf{9}$ & minus & $\mathbf{9}$ & & 4 & plus & 2 & minus & 4 \\
& 2 & plus & 6 & minus & 5 & & 6 & plus & 0 & minus & 5 \\
\hline & & & & & & & & & & & \\
\hline Q7 & 0 & plus & 5 & minus & 0 & Q10 & 9 & plus & 6 & minus & 3 \\
& $\mathbf{6}$ & plus & $\mathbf{8}$ & minus & $\mathbf{0}$ & & 3 & plus & 0 & minus & 1 \\
& 3 & plus & 2 & minus & 8 & & 0 & plus & 2 & minus & 3 \\
& 2 & plus & 2 & minus & 8 & & $\mathbf{7}$ & plus & $\mathbf{9}$ & minus & $\mathbf{1}$ \\
\hline
\end{tabular}

The four Simple 8 questions use the same format as that given in figure 2 together with the following numbers:

\begin{tabular}{cccccccccccc}
\hline Q2 & 2 & plus & 0 & minus & 8 & Q5 & 2 & plus & 8 & minus & 9 \\
& 6 & plus & 3 & minus & 5 & & $\mathbf{4}$ & plus & $\mathbf{9}$ & minus & $\mathbf{2}$ \\
& 5 & plus & 8 & minus & 9 & & 1 & plus & 2 & minus & 9 \\
& 7 & plus & 2 & minus & 4 & & 2 & plus & 8 & minus & 1 \\
& 7 & plus & 4 & minus & 9 & & 4 & plus & 5 & minus & 0 \\
& 5 & plus & 1 & minus & 0 & & 4 & plus & 7 & minus & 4 \\
& $\mathbf{6}$ & plus & $\mathbf{3}$ & minus & $\mathbf{0}$ & & 6 & plus & 4 & minus & 9 \\
& 9 & plus & 0 & minus & 9 & & 4 & plus & 6 & minus & 0 \\
\hline & & & & & & & & & & & \\
\hline Q8 & 0 & plus & 4 & minus & 4 & Q11 & 5 & plus & 8 & minus & 9 \\
& 2 & plus & 0 & minus & 8 & & 3 & plus & 3 & minus & 4 \\
& 2 & plus & 6 & minus & 3 & & 5 & plus & 1 & minus & 4 \\
& 5 & plus & 1 & minus & 7 & & 4 & plus & 4 & minus & 8 \\
& 0 & plus & 3 & minus & 1 & & 4 & plus & 5 & minus & 3 \\
& $\mathbf{8}$ & plus & $\mathbf{6}$ & minus & $\mathbf{5}$ & & 4 & plus & 5 & minus & 3 \\
& 3 & plus & 5 & minus & 2 & & 1 & plus & 8 & minus & 6 \\
& 0 & plus & 7 & minus & 8 & & $\mathbf{9}$ & plus & $\mathbf{7}$ & minus & $\mathbf{9}$ \\
\hline
\end{tabular}

The four Complex 4 questions use the same format as that given in figure 3 together with the following numbers:

\begin{tabular}{cccccccccccccccc}
\hline Q3 & 7 & plus & 2 & minus & 5 & minus & 1 & Q6 & $\mathbf{6}$ & plus & $\mathbf{2}$ & minus & $\mathbf{0}$ & minus & $\mathbf{3}$ \\
& $\mathbf{4}$ & plus & $\mathbf{6}$ & minus & $\mathbf{3}$ & minus & $\mathbf{2}$ & & 2 & plus & 4 & minus & 2 & minus & 6 \\
& 7 & plus & 0 & minus & 2 & minus & 9 & & 1 & plus & 7 & minus & 5 & minus & 5 \\
& 6 & plus & 4 & minus & 7 & minus & 4 & & 6 & plus & 5 & minus & 4 & minus & 5 \\
\hline & & & & & & & & & & & & & & \\
\hline Q9 & 7 & plus & 3 & minus & 4 & minus & 8 & Q12 & 0 & plus & 7 & minus & 1 & minus & 5 \\
& 2 & plus & 9 & minus & 9 & minus & 9 & & 0 & plus & 6 & minus & 1 & minus & 7 \\
& 5 & plus & 1 & minus & 3 & minus & 0 & & $\mathbf{4}$ & plus & $\mathbf{1}$ & minus & $\mathbf{0}$ & minus & $\mathbf{2}$ \\
& $\mathbf{3}$ & plus & $\mathbf{9}$ & minus & $\mathbf{0}$ & minus & $\mathbf{7}$ & & 2 & plus & 5 & minus & 6 & minus & 4 \\
\hline
\end{tabular}




\section{Suggested points for reflection and debrief after the experiment (with typical answers)}

We suggest instructors to use the following text and questions as the basis for the reflection session immediately after the experiment. These can be distributed as standard lecture notes or as handouts after the experiment if helpful.

"In previous lectures, we have established a deeper understanding of the standard (or 'neoclassical') model of consumer choice. However, as we shall see, in some settings, the assumptions of the standard model seem too strong. As a result, the predictions of the standard model do not always provide a close approximation to actual behaviour.

Q. Do you think you always picked the 'correct' answer in the experiment?

Q. When do you think you were more likely to pick an 'incorrect' answer?

(Typical answers will indicate that students find the decisions harder when there were more options and when the options were more complex.)

One explanation for anomalies in these situations is 'bounded rationality' where individuals find it optimal to i) only consider some of the options, or ii) to use 'heuristics' or 'rules of thumb' when making their calculations and selections, in order to conserve cognitive resources.

Q. When faced with a tough decision in the experiment, how did you decide what to select? Did you consider all the options? What 'rules of thumb' did you employ for your calculations and selections?

(Typical answers will indicate that students focussed on answers near the top of the list because they didn't have time to study them all (as consistent with the primacy effect), or on answers with high starting numbers (as consistent with the left-digit-bias). 
Q. Many individuals will exhibit a bias towards certain types of options. Can you think which types of options might have been more likely to have been picked in the experiment?

(Typical answers will indicate that the options with big starting numbers or the options near the top may be picked more often.)

Q. If consumers exhibit these forms of biases, how do you think businesses would want to adapt their business strategies to exploit consumers?

(Typical answers will indicate that businesses will want their products near the top of lists (e.g. search engines) and that they may want put the most attractive or profitable information first to best use consumer's attention.)

\section{Tips for instructors to construct the results}

If the instructor is either using a clicker technology or an alternative paper-based approach with OMR technology, then the raw data of the responses per question can often be easily retrieved as a spreadsheet. For each question, such results typically show the overall response rate, the percentage of responses that selected each option, and the number of students that selected each option.

With this in hand, Table 1 can be easily calculated by identifying the correct answer for each question using our information above and then averaging the appropriate percentages across the relevant questions. Similarly, Table 3 can also be constructed very easily by averaging the appropriate percentages for options A-D. The construction of Table 2 requires a little more work. For each incorrect option, one needs to construct the following columns on a spreadsheet: the value of the first digit, the value of the second digit, the value of the last digit, and the number of students that selected that option. To assess the relationship between particular digits 
and student choice, one can then find the pairwise correlations between each of the first three columns with the last column. 


\section{TABLES}

TABLE 1: Summary of Results

\begin{tabular}{lccc} 
& $\begin{array}{c}\text { Total } \\
\text { Responses }\end{array}$ & $\begin{array}{c}\text { Response } \\
\text { Rate }(\%)\end{array}$ & $\begin{array}{c}\text { Incorrect } \\
\text { Responses (\%) }\end{array}$ \\
\hline All questions & 1325 & 88.3 & 23.3 \\
Round 1 & 301 & 80.3 & 30.6 \\
Round 2 & 346 & 92.3 & 12.9 \\
Round 3 & 351 & 93.6 & 19.0 \\
Round 4 & 327 & 87.2 & 30.6 \\
& & & \\
Simple 4 & 482 & 96.4 & 2.8 \\
Simple 8 & 404 & 80.8 & 48.2 \\
Complex 4 & 439 & 92.8 & 18.9 \\
\hline
\end{tabular}

TABLE 2: Analysis of Left and Right Digit Bias

Correlation between the number of incorrect choices and ...

\begin{tabular}{lc}
\hline$\ldots$ value of first digit & 0.19 \\
$\ldots$ value of second digit & 0.06 \\
$\ldots$ value of last digit & -0.34 \\
\hline
\end{tabular}

TABLE 3: Analysis of Primacy and Recency Effects

\begin{tabular}{lc} 
& Average \% of responses \\
\hline Option A & 28.00 \\
Option B & 24.85 \\
Option C & 26.20 \\
Option D & 20.95 \\
\hline
\end{tabular}




\section{FIGURES}

FIGURE 1: An Example Question (Simple 4)

Which option has the highest value?
A. Three plus one minus two
B. Seven plus two minus five
C. Two plus five minus two
D. One plus seven minus six

FIGURE 2: An Example Question (Simple 8)

Which option has the highest value?
A. Zero plus zero minus zero
B. Nine plus seven minus seven
C. One plus three minus seven
D. Nine plus one minus five
E. Seven plus eight minus nine
F. Three plus nine minus eight
G. Nine plus two minus three
H. Three plus six minus one

FIGURE 3: An Example Question (Complex 4)

Which option has the highest value?
A. Zero plus zero minus zero minus two
B. Nine plus seven minus seven minus four
C. One plus three minus seven minus one
D. Nine plus one minus five minus five 